\title{
PRATIQUES ET APPORTS DE LA QPC EN DROIT DE LA SANTÉ (PAQS)
}

\author{
Responsable scientifique Marie Mesnil, Pascal Caillaud, Marion Del Sol, Josépha \\ Dirringer, Suzanne Poulot, Évelyne Serverin
}

Conseil constitutionnel | «Titre VII »

2020/octobre Hors série | pages 221 à 237

DOI 10.3917/tvii.hs.001.0221

Article disponible en ligne à l'adresse :

https://www.cairn.info/revue-titre-vii-2020-octobre-page-221.htm

Distribution électronique Cairn.info pour Conseil constitutionnel.

(C) Conseil constitutionnel. Tous droits réservés pour tous pays.

La reproduction ou représentation de cet article, notamment par photocopie, n'est autorisée que dans les limites des conditions générales d'utilisation du site ou, le cas échéant, des conditions générales de la licence souscrite par votre établissement. Toute autre reproduction ou représentation, en tout ou partie, sous quelque forme et de quelque manière que ce soit, est interdite sauf accord préalable et écrit de l'éditeur, en dehors des cas prévus par la législation en vigueur en France. Il est précisé que son stockage dans une base de données est également interdit. 


\title{
Partie 4 - La QPC, 10 ans de jurisprudence
}

\section{Pratiques et Apports de la QPC en droit de la Santé (PAQS)}

\author{
Marie MESNIL, Responsable scientifique \\ Maîtresse de conférences à l'Université de Rennes 1, Chercheure à l'IODE (UMR CNRS 6262)
}

Pascal CAILLAUD

Chargé de recherche CNRS, Laboratoire droit et changement social (UMR-CNRS 6297)

Marion DEL SOL

Professeure à l'Université de Rennes 1, Chercheuse à I'IODE (UMR CNRS 6262)

Josépha DIRRINGER

Maîtresse de conférences à l'Université de Rennes 1, Chercheure à l'IODE (UMR CNRS 6262)

\section{Suzanne POULOT}

Ingénieure d'études

\section{Evelyne SERVERIN}

Directrice de recherche CNRS

Instaurée par la réforme constitutionnelle du 23 juillet 2008, la question prioritaire de constitutionnalité (QPC) a significativement modifié le procès et l'architecture juridictionnelle française' ${ }^{1}$. Entre le $1^{\text {er }}$ mars 2010 et le 30 juin 2019, le Conseil constitutionnel s'est prononcé sur 706 QPC alors que, sur la même période, il a rendu 187 décisions résultant d'une saisine a priori ${ }^{2}$. Il ne s'agit pourtant que de la pratique la plus visible (« la partie émergée de l'iceberg ») dans la mesure où un très grand nombre de QPC ne passe pas l'examen de recevabilité des juridictions du fond devant lesquelles elles sont posées ou encore celui opéré par

1. Emmanuel Cartier, La question prioritaire de constitutionnalité : étude sur le réagencement du procès et de l'architecture juridictionnelle française, Université de Lille 2, Mission de recherche Droit et justice, 2012.

2. Conseil constitutionnel, «Bilan statistique au 30 juin $2019 »$, Titre VII. 
les juridictions suprêmes de l'ordre judiciaire ou administratif ${ }^{3}$.

En associant les expertises de chercheurs en droit de la santé, droit de la santé au travail et droit de la protection sociale et de sociologues du droit, cette recherche visait à étudier à la fois les pratiques et les apports de la QPC en droit de la santé, entendu au sens large comme comprenant également le droit de la protection sociale et le droit de la santétravail. Il s'agissait plus particulièrement de comprendre de quelle manière la question prioritaire de constitutionnalité a été intégrée en droit de la santé à la fois comme moyen de droit, mobilisé par les justiciables et modifiant les pratiques judiciaires, et comme source du droit, modifiant de manière substantielle les règles de droit.

La délimitation de l'objet de la recherche correspond à l'ensemble des thématiques du droit de la santé : il ne s'agit pas d'étudier les QPC qui invoquent le droit à la santé - protégé par le onzième alinéa du Préambule de la Constitution 1946 - mais toutes celles qui mettent en cause une disposition législative qui relève du champ matériel du droit de la santé. L'intérêt de cette approche par la matière permet de couvrir des thématiques nombreuses, dont la mise en œuvre relève de juridictions spécialisées de l'ordre judiciaire et des juges de droit commun de l'ordre judiciaire et de l'ordre administratif. Les domaines considérés du droit de la santé ont plus spécifiquement trait à l'organisation du système de santé, aux droits des malades et à la bioéthique, aux personnels et établissements de santé, aux produits de santé, y compris dans les dimensions relatives à la responsabilité et à l'indemnisation, mais aussi à la protection sociale (assurance maladie, régime des accidents du travail et des maladies professionnelles...) et à la santé au travail. Les thèmes de la santé au travail et du droit de la protection sociale ont pris une place considérable avec, d'un côté, la reconnaissance d'une obligation de sécurité de résultat, à peine atténuée, mise à la charge de l'employeur', et de l'autre une universalisation de la couverture maladie de base ${ }^{5}$, assortie d'une obligation pour l'entreprise d'assurer une couverture santé complémentaire aux salariés ${ }^{6}$. En outre, le contentieux en droit de la protection sociale présente des spécificités importantes aussi bien en termes d'organisation (juges non professionnels) que de procédure (orale, sans ministère d'avocat obligatoire, avec la possibilité d'être représenté par un défenseur syndical devant le conseil de prud'hommes). Les pratiques de la QPC devant ces juridictions spécialisées - et notamment devant les pôles sociaux mis en place depuis le $1^{\text {er }}$ janvier 2019 au sein des tribunaux de grande instance (TGI) ${ }^{7}$ - ont ainsi pu être étudiées afin de rendre compte de la manière dont les justiciables se saisissent de ce nouveau moyen de droit et dont les juges mobilisent des décisions QPC rendues comme source du droit.

Le projet de recherche poursuivait principalement deux finalités qui se sont réciproquement nourries : d'une part, étudier les pratiques de la QPC devant les juridictions spécialisées qui traitent du contentieux du droit la protection sociale et de la santé-travail (A) et, d'autre part, établir un bilan

3. Agnès Roblot-Troizier, « La QPC, le Conseil d'État et la Cour de cassation », Nouveaux Cahiers du Conseil constitution$n e l, \mathrm{n}^{\circ} 40$, juin 2013.

4. Voir not. Cass. soc., 28 fév. 2002, $\mathrm{n}^{\circ} 00-10.051, \mathrm{n}^{\circ} 99-18.389, \mathrm{n}^{\circ} 00-11.793, \mathrm{n}^{\circ} 99-18.390, \mathrm{n}^{\circ}$ 99-21.255, $\mathrm{n}^{\circ}$ 99-17.201; Cass. soc., 11 avril 2002, n 00-16535 et Cass. soc., 28 fév. 2006, $n^{\circ}$ 05-41555.

5. Instauration de la Protection Universelle Maladie (PUMA) par la loi $\mathrm{n}^{\circ}$ 2015-1702 du 21 décembre 2015 de financement de la sécurité sociale pour 2016, voir not. Didier Tabuteau, « La protection universelle maladie (PUMA) : une transfiguration législative de l'assurance maladie (première partie) », RDSS, 2015, p. 1058 et « La protection universelle maladie (PUMA) : une rationalisation inachevée du financement de la sécurité sociale (seconde partie) », RDSS, 2016, p. 131.

6. Accord national interprofessionnel (ANI) du 11 janvier 2013, loi n 2013-504 du 14 juin 2013 relative à la sécurisation de l'emploi et décret n ${ }^{\circ}$ 2014-1025 du 8 septembre 2014 relatif aux garanties d'assurance complémentaire santé des salariés mises en place en application de l'article L. 911-7 du Code de la sécurité sociale.

7. Loi n $2016-1547$ du 18 novembre 2016 de modernisation de la justice du XXI ${ }^{e}$ siècle. 
jurisprudentiel thématique, couvrant cette foisci l'ensemble du droit de la santé lato sensu, afin de saisir les apports substantiels de la QPC à la matière $(B)$.

\section{A. Les pratiques de la QPC dans le champ du droit de la protection sociale et de la santé-travail}

Une étude de sociologie juridique des pratiques de la QPC devant les juridictions spécialisées en droit de la protection sociale et de la santé-travail a été menée. Cette étude s'est déroulée en deux temps : d'une part, une étude qualitative de la pratique de la QPC par les juges au sein des juridictions spécialisées en droit de la protection sociale et de la santé-travail (1) et, d'autre part, une analyse quantitative des QPC posées et/ou citées devant les cours d'appel en matière civile (2).

\section{Analyse qualitative de la pratique de la QPC en droit de la protection sociale et de la santé-travail}

L'étude de sociologie juridique sur la pratique de la QPC par les juges au sein des juridictions spécialisées en droit de la protection sociale et de la santé-travail visait à déterminer dans quelle mesure la QPC a eu une incidence sur leur activité juridictionnelle.

Les pôles sociaux des TGI reprennent la composition particulière des juridictions spécialisées en droit de la protection sociale et intègrent ainsi un magistrat professionnel tandis que les conseils de prud'hommes sont composés uniquement de juges non professionnels. Au regard de ces spécificités, il a été étudié, d'un côté, l'incidence de la QPC sur l'activité juridictionnelle des magistrats des pôles sociaux des TGI et, de l'autre, la manière dont les organisations syndicales se sont saisies, dans le champ du droit de la santé et notamment de la santé-travail, de ce nouveau moyen de droit devant les juridictions sociales (formation des conseillers prud'hommes, des défenseurs syndicaux et stratégie contentieuse).

\subsection{Létude des pratiques de la QPC devant les juridictions sociales}

\subsubsection{Méthodologie}

L'étude des pratiques de la QPC devant les juridictions sociales du premier degré a été réalisée en mobilisant deux types d'outils d'enquête : d'un côté, des entretiens semi-directifs avec les principaux acteurs juridictionnels des pôles sociaux (magistrats, assesseur, procureur) au sein des TGI ; de l'autre, un questionnaire auto-administré en ligne, envoyé aux juges et assesseurs des pôles sociaux ainsi qu'aux conseillers prud'hommes salariés.

Les entretiens semi-directifs ont été utilisés à titre informatif afin de prendre la mesure du contentieux QPC devant les juridictions de sécurité sociale. Il s'agissait d'analyser la pratique de la QPC par les juges des juridictions spécialisées en droit de la protection sociale et d'appréhender leur perception de l'objet et de l'utilité de la QPC au regard de l'usage qui en est fait par les justiciables. Ils ont été menés à partir d'un guide d'entretien élaboré collectivement et concentré autour de trois thématiques : $1^{\circ}$ ) la pratique juridictionnelle de la $\mathrm{QPC}, 2^{\circ}$ ) la perception des évolutions engendrées par la QPC et enfin $3^{\circ}$ ) la perception de l'objet de la QPC. Ces thématiques sont reprises dans le questionnaire.

$\mathrm{Au}$ total, huit entretiens ont été réalisés : cinq entretiens avec des magistrate's de pôles sociaux, un autre avec un ancien assesseur de pôle social et le dernier avec un membre du parquet civil. Il a été choisi de privilégier les entretiens avec des 
magistrat $\cdot e \cdot s$ car le magistrat du pôle social statue seul sur les demandes de transmission de QPC.

Le questionnaire auto-administré a été diffusé par courriel auprès de l'ensemble des greffes et secrétariats des TGI à destination des magistrats et assesseurs des pôles sociaux et, par le biais des organisations syndicales, auprès de leurs conseillers prud'hommes. En définitive, 55 réponses au questionnaire ont été reçues, le taux de réponse des conseillers prud'hommes étant légèrement supérieur à celui des magistrats et assesseurs des pôles sociaux.

\subsubsection{Analyse des entretiens}

Les résultats des entretiens ont été complétés et prolongés par ceux obtenus par le biais des questionnaires. Ils ont révélé que la pratique de la QPC par les magistrat'e-s des pôles sociaux est majoritairement dominée par le contentieux dit des "Libérés de la sécurité sociale », un mouvement mené par un certain nombre de professions libérales - professionnels de santé ou indépendants - qui conteste l'affiliation obligatoire à un régime de protection sociale. Ces $\mathrm{QPC}$, qui font partie d'un ensemble plus large de manœuvres dilatoires utilisées par ces justiciables, ne sont pas considérées comme pertinentes par les magistrat'e-s puisqu'elles remettent en cause le principe même des régimes de protection sociale fondés sur des mécanismes de solidarité.

En dépit du nombre significatif de QPC auquel les magistrats des pôles sociaux sont confrontés, la mise en place de ce nouveau moyen de droit n'a pas modifié de manière importante leur pratique juridictionnelle quotidienne. À la marge, lorsqu'ils doivent répondre aux demandes de transmission de $\mathrm{QPC}$, et notamment à celles qui ne relèvent pas du contentieux des "Libérés de la sécurité sociale ", le dispositif oblige les magistrats à s'intéresser aux contentieux constitutionnels. En particulier, les QPC posées - lorsqu'elles sont recevables - leur demandent de se mettre à jour, plus ou moins régulièrement, sur les décisions rendues par la Cour de cassation, le Conseil d'État et le Conseil constitutionnel en la matière.

La spécificité de la procédure devant les pôles sociaux et la technicité de la matière sont des éléments explicatifs qui ont été mis en avant par les magistrate's pour comprendre le manque de pertinence des questions posées. Les entretiens ont aussi révélé que l'appréciation, par le juge du premier degré, du caractère sérieux de la question posée n'était pas évidente. En effet, aucun d'entre eux n'a été en mesure d'apporter une définition claire de ce critère d'appréciation qui leur permet toutefois d'évacuer les QPC posées à des fins dilatoires. Le magistrat e qui a tenu, sur les critères de recevabilité, les propos les plus élaborés lors des entretiens a demandé et bénéficié d'une formation spécifique à la QPC. Pour les autres, la nécessité d'une formation ne s'est pas imposée à eux compte tenu du faible nombre de QPC posées au regard de la masse du stock de dossiers à traiter, du caractère très technique du contentieux du droit de la sécurité sociale et de la relative simplicité de la procédure en matière de QPC.

L'utilité de la QPC est perçue de manière assez différente par les conseillers prud'hommes et par les magistrat'e's des pôles sociaux, ce qui pourrait s'expliquer par la pratique plus importante de la QPC par ces derniers. Les conseillers prud'hommes ont, dans l'ensemble, une vision plutôt positive de la QPC même s'ils n'y ont pas été confrontés. En effet, sur les 29 conseillers ayant répondu au questionnaire, seuls deux ont eu à en connaître. À la question « à quoi sert la QPC ? », les conseillers prud'hommes répondent majoritairement que le dispositif doit permettre de protéger les droits et libertés fondamentaux des justiciables. De fait, les magistrate's interrogés par le bais des entretiens et du questionnaire mettent également en avant, et de manière positive, la fondamentalisation du droit qu'elle permet. Toutefois, au regard de leur pratique de la QPC, ils concluent que le dispositif ne répond qu'imparfaitement à cet objectif. 


\subsubsection{Conclusion}

Si l'utilité de la QPC n'est pas remise en cause par les magistrat·e's interrogé'e-s dans le cadre des entretiens semi-directifs, les répondants au questionnaire hsont beaucoup plus réservés sur l'opportunité du dispositif. Cette différence peut s'expliquer par la forme même du questionnaire qui ne permet, notamment en raison de l'anonymat, que des réponses courtes et plus tranchées. À l'inverse, le format des entretiens a permis aux magistrate's $\mathrm{s}$ interrogée's d'expliciter plus longuement leur pensée. L'usage dilatoire des QPC semble moins influencer les magistrat'e-s qui ont été entendus que ceux qui ont répondu au questionnaire. En outre, les magistrat'e's ayant répondu au questionnaire soulèvent également une réserve émise par un des magistrat·e's interrogé·e's, à savoir l'insécurité juridique induite par ce contrôle a posteriori de dispositions législatives.

\subsection{Les pratiques syndicales de la QPC devant les juridictions sociales}

\subsubsection{Méthodologie}

Les conseils de prud'hommes ont la particularité d'être paritaires et d'être composés d'un nombre égal de salariés et d'employeurs. Ils sont composés uniquement de juges non professionnels, dont la formation représente un enjeu important. Les organisations syndicales n'interviennent pas seulement dans le contentieux prud'homal à travers les conseillers issus de leurs rangs mais aussi par le biais des défenseurs syndicaux. C'est pourquoi il a été jugé pertinent d'interroger les principales organisations syndicales sur l'usage qu'elles font de la QPC.

Dans le cadre de l'étude des pratiques syndicales de la QPC devant les juridictions sociales, ont été étudiées la formation des conseillers prud'hommes mais également l'intégration de la QPC comme élément de stratégie judiciaire des organisations syndicales. Il s'agissait d'apprécier la place prise par la QPC dans la formation initiale et continue des conseillers prud'hommes et l'usage que pouvaient en faire les organisations syndicales. Pour ce faire, l'École nationale de la magistrature et six Instituts du travail (Paris, Strasbourg, Bordeaux, Grenoble, Nancy et Rennes) ont été contactés afin de déterminer si une formation spécifique à la QPC était dispensée aux conseillers prud'hommes. Parallèlement, un questionnaire a été envoyé à quatre confédérations syndicales de salariés, notamment aux personnes responsables du service juridique au sein de ces confédérations : la CFDT, la CFE-CGC, la CGT et la CGT-FO. Seule la CGT-FO n'a pas répondu à notre sollicitation. Le questionnaire, envoyé par mail, comportait six questions centrées sur leur pratique de la QPC en matière de droit de la santé-travail et les actions de formation susceptibles de sensibiliser les équipes syndicales à la QPC.

\subsubsection{Analyse des éléments recueillis}

Dans la formation initiale comme dans la formation continue des conseillers prud'hommes, une faible place est accordée à la QPC. Des informations recueillies auprès de l'ENM, il ressort que le sujet de la QPC n'a pas été abordé spécifiquement dans le cadre de la formation initiale des conseillers prud'hommes. De même, aucune formation prud'homale récente n'a, semble-t-il, été dédiée au seul sujet de la QPC au sein des six Instituts du travail sollicités. En revanche, l'ENM révise à l'heure actuelle le contenu du volet « e-formation » et, à ce titre, a élaboré une fiche technique spécifique sur le traitement de la QPC devant le conseil de prud'hommes. Le document n'est pas encore à la disposition des conseillers mais devrait l'être d'ici l'été 2020.

S'agissant des questionnaires adressés par mail aux organisations syndicales, il ressort des réponses qu'aucune confédération n'a initié de QPC dans le domaine de la santé-travail. De fait, si toutes les confédérations la prennent en compte et s'interrogent, en cas de contentieux, sur la mobilisation de cet instrument, il demeure, malgré tout, une certaine réticence à l'utiliser. C'est notamment le 
cas de la CFDT et de la CGT qui sont généralement méfiantes à l'égard de l'utilisation de la QPC dans leur stratégie syndicale compte-tenu de la manière dont le juge constitutionnel exerce, selon elles, son contrôle de constitutionnalité en matière sociale. En effet, elles estiment que le Conseil constitutionnel, lorsqu'il est saisi de l'interprétation des droits sociaux, invoque trop souvent le motif d'intérêt général pour justifier des atteintes portées à des droits constitutionnellement protégés et qu'en toute hypothèse, son contrôle demeure trop formel et abstrait. En outre, la CGT souligne que les décisions du Conseil constitutionnel sont généralement peu favorables aux droits des travailleurs alors que le droit et les institutions internationales leur semblent plus protecteurs des droits fondamentaux des salariés.

\subsubsection{Conclusion}

Pour expliquer la faible place occupée par la QPC dans la formation initiale et continue des conseillers prud'hommes, plusieurs hypothèses peuvent être avancées. La première repose sur des considérations d'ordre pratique. En effet, former des juges non professionnels à la fois au droit matériel qu'ils auront à appliquer et à l'exercice de fonctions juridictionnelles est une véritable gageure. Dès lors, des arbitrages sont nécessairement effectués, ce qui conduirait à laisser peu d'espace pour aborder la QPC. En outre, le contentieux devant le conseil de prud'hommes est peu propice au déploiement de stratégies judiciaires mobilisant, voire instrumentalisant, la QPC. En effet, il s'agit d'un contentieux relatif aux relations individuelles de travail massivement initié par le salarié et poursuivant très souvent une fin indemnitaire. L'analyse de certains contentieux QPC en droit social lato sensu semble confirmer le point de vue développé par un ancien membre du Conseil constitutionnel selon lequel la QPC n'est pas un recours individuel mais un recours collectif « destiné à faire sauter une législation qui contrarie ou heurte les valeurs d'un groupe social $\|^{8}$. Dès lors, la situation très concrète du demandeur à une instance prud'homale ne doit guère l'inviter (ou inviter celui qui exerce sa défense) à envisager de soulever une QPC dont la transmission retarderait l'issue du litige. En d'autres termes, les caractéristiques mêmes des litiges prud'homaux et des enjeux individuels seraient peu favorables à un usage instrumentalisé de la QPC à des fins de défense de valeurs intéressant un collectif ou un groupe social.

S'agissant de l'absence de QPC soulevée par les défenseurs syndicaux dans le domaine du droit de la santé-travail, elle peut s'expliquer par le fait que la QPC a plutôt été utilisée dans le champ des relations collectives de travail où de nombreuses réformes sont intervenues ces dernières années. En outre, ces réformes ont intéressé les organisations syndicales en tant que telles et non pas seulement en tant que représentantes des intérêts des salariés. Enfin, une autre explication tiendrait à l'organisation interne des confédérations syndicales, le service chargé des questions de santé étant en effet souvent distinct du service juridique. Les premiers seraient davantage tournés vers la prévention dans le domaine de la santé que vers la réparation, mettant ainsi à distance le recours au droit. Quant aux seconds, ils seraient davantage tournés vers les questions de droit du travail, spécialement le droit des relations collectives de travail, notamment du fait de leur formation initiale.

\section{Analyse de la pratique de la QPC devant les cours d'appel en droit de la protection sociale et de la santé-travail}

\subsection{Méthodologie}

Après la signature d'une convention avec le service de documentation, des études et du rapport (SDER) de la Cour de cassation, il a été possible

8. Olivier Dutheillet de Lamothe, « La QPC en droit du travail. Point de vue du juge constitutionnel », Dr. soc. 2015 , p. 480. 
d'accéder à la base de données JuriCA qui rassemble tous les arrêts civils rendus par les juridictions du second degré de l'ordre judiciaire depuis 2007.

La consultation de JuriCA visait à prendre la mesure de la pratique de la QPC devant les juridictions d'appel en droit de la protection sociale et, ainsi, à prolonger les études qualitatives menées auprès des juridictions sociales.

Après plusieurs explorations, il a été choisi de délimiter un corpus de décisions selon deux critères : concernant le champ matériel, le mot-clé « code de la sécurité sociale » a été retenu ainsi que la mention du moyen de droit « question prioritaire de constitutionnalité ». La forme développée correspond à la formule retenue lorsqu'une « question prioritaire de constitutionnalité »a été posée devant la juridiction ou lors de la procédure tandis que la forme abrégée QPC renvoie aux décisions des cours d'appel qui mentionnent des décisions QPC rendues par le Conseil constitutionnel. Le mot-clé « QPC » a ainsi été retenu pour constituer un autre corpus permettant de saisir de quelle manière la QPC modifie le droit matériel au point de devenir une source de droit.

Le corpus constitué par une requête [« question prioritaire de constitutionnalité » et « code de la sécurité sociale »] comprend 2003 arrêts rendus par des cours d'appel. Il correspond aux décisions d'appel mentionnant une question prioritaire de constitutionnalité posée par un justiciable devant la juridiction d'appel ou en amont dans la procédure. Il permet ainsi d'accéder à l'usage fait par les justiciables de la question prioritaire de constitutionnalité comme moyen de droit lors d'une procédure contentieuse en droit de la sécurité sociale.

\subsection{Analyse des données}

Après extraction du corpus à partir de JuriCA, nous avons procédé à de nouvelles lectures et explorations afin de dresser une cartographie des usages faits de la QPC par les justiciables.

Dans un premier temps, toutes les décisions du corpus qui mentionnent la décision $\mathrm{n}^{\circ} 2010-8$ QPC du 18 juin 2010, Époux L., en utilisant l'expression « question prioritaire de constitutionnalité » et le numéro de la décision ou la date ont été retirées, ce qui représente 294 éléments. L'exploitation détaillée a porté sur un corpus de 1710 décisions. Nous avons alors identifié les grandes lignes du contentieux, puis procédé à des analyses successives sur chacun des groupes thématiques.

Au regard des entretiens et questionnaire que nous avons réalisés, il est apparu que le contentieux en lien avec le recouvrement des cotisations sociales occupait une place importante. Nous avons donc isolé toutes les questions prioritaires de constitutionnalité en lien avec le contentieux du recouvrement de cotisations sociales. Pour cela, nous avons identifié plusieurs mots-clés : « opposition à contrainte ", « recouvrement ", «URSSAF », « cotisations », « cotisation » et « RSI ». Le motclé « redressement » a été exclu à cause de l'expression « redressement judiciaire » dans laquelle il apparaît fréquemment. Les tableaux ci-dessous décomptent les occurrences des mots-clés dans le corpus. 
Tableau 1 - Le thème du recouvrement des cotisations

\begin{tabular}{l|l} 
Mot-clé & Nombre de décisions comportant les mots-clés (sur 1710) \\
\hline URSSAF & 758 \\
\hline Opposition à contrainte & 552 \\
\hline Recouvrement & 783 \\
\hline Cotisation / Cotisations & $1170 / 1162$ \\
\hline RSI & 397 \\
\hline & $\begin{array}{l}\text { Total = } 1379 \\
\text { (après suppression des doublons) }\end{array}$ \\
\hline
\end{tabular}

Sur cet ensemble de 1379 décisions, nous avons effectué d'autres recherches par mot-clé :

\begin{tabular}{l|l}
\hline Mot-clé & Nombre de décisions comportant les mots-clés (sur 1 379) \\
\hline " Refus de transmission " & 252 \\
\hline " Ordonne la transmission " & 26 \\
\hline " Amende civile " & 278 \\
\hline " Dilatoire " & 295 \\
\hline $\begin{array}{l}\text { " Amende civile " ou } \\
\text { "Dilatoire " }\end{array}$ & 360
\end{tabular}

En retirant du corpus toutes les décisions relatives au contentieux du recouvrement des cotisations sociales, on identifie 330 décisions qui ont fait l'objet d'une analyse séparée (tableau 2 ci-dessous).

Tableau 2 - Les autres thèmes

\begin{tabular}{l|l}
\hline Mot-clé & Nombre de décisions comportant les mots-clés (sur 330) \\
\hline " Retraite " & 100 \\
\hline " Refus de transmission " & 18 \\
\hline "Ordonne la transmission » & $\begin{array}{l}9 \text { dont } 5 \text { qui " a ordonné la transmission " } \\
\text { et } 4 \text { qui « ordonne la transmission » }\end{array}$ \\
\hline
\end{tabular}

Les 18 décisions de refus de transmission et les 4 décisions qui ordonnent la transmission ont fait l'objet d'une analyse plus précise (tableaux 3 et 4 ci-dessous).

Parmi les décisions de transmission de la QPC, il apparaît que le principe d'égalité est particulièrement mobilisé comme droits et libertés garantis par la Constitution et ce dans le contexte de dispositions législatives variées.

Les décisions de refus de transmission sont, quant à elles, motivées par l'absence de caractère sérieux de la question ou encore l'irrecevabilité de celle-ci du fait du caractère d'ordre réglementaire des dispositions dont la constitutionnalité est contestée. 
Tableau 3 - Analyse des décisions ordonnant la transmission de la question prioritaire de constitutionnalité

\begin{tabular}{|c|c|c|c|}
\hline Arrêts & $\begin{array}{l}\text { Motif de } \\
\text { transmission }\end{array}$ & $\begin{array}{l}\text { Dispositions législatives } \\
\text { en cause }\end{array}$ & $\begin{array}{l}\text { Droits et liberté } \\
\text { invoqués }\end{array}$ \\
\hline $\begin{array}{l}\text { Versailles } \\
16 \text { février } 2010 \\
n^{\circ} 10 / 00015\end{array}$ & $\begin{array}{l}\text { Caractère } \\
\text { sérieux }\end{array}$ & $\begin{array}{l}\text { Article L. } 351-13 \text { du Code } \\
\text { de la sécurité sociale : } \\
\text { pension vieillesse assortie } \\
\text { d'une majoration lorsque } \\
\text { le conjoint à charge n'est } \\
\text { pas bénéficiaire d'un } \\
\text { avantage, quel que soit le } \\
\text { montant de l'avantage }\end{array}$ & $\begin{array}{l}\text { Principe d'égalité : ar- } \\
\text { ticle } 34 \text { de la Consti- } \\
\text { tution et article } 6 \text { de } \\
\text { la DDHC }\end{array}$ \\
\hline $\begin{array}{l}\text { Nancy } \\
4 \text { novembre } 2016 \\
n^{\circ} 16 / 02029\end{array}$ & $\begin{array}{l}\text { Caractère } \\
\text { sérieux }\end{array}$ & $\begin{array}{l}\text { Article L. 323-1 du Code } \\
\text { de la sécurité sociale : } \\
\text { limitation à } 360 \text { indemni- } \\
\text { tés journalières par } \\
\text { trois ans }\end{array}$ & $\begin{array}{l}\text { Principe d'égalité : ar- } \\
\text { ticle } 1^{\text {er }} \text { du préambule } \\
\text { de la Constitution de } \\
\text { 1958, article } 6 \text { de la } \\
\text { DDHC et article } 7 \\
\text { de la DUDH }\end{array}$ \\
\hline $\begin{array}{l}\text { Rennes } \\
25 \text { juin } 2014 \\
n^{\circ} 13 / 07522\end{array}$ & $\begin{array}{l}\text { Question } \\
\text { qui n'est pas } \\
\text { nouvelle } \\
\text { mais pré- } \\
\text { sentant un } \\
\text { caractère } \\
\text { sérieux }\end{array}$ & $\begin{array}{l}\text { Dispositif amiante : loi de } \\
\text { financement de la sécurité } \\
\text { sociale pour } 2012\end{array}$ & $\begin{array}{l}\text { Principe général } \\
\text { d'égalité }\end{array}$ \\
\hline $\begin{array}{l}\text { Aix-en-Provence } \\
18 \text { novembre } 2010 \\
n^{\circ} 10 / 08865\end{array}$ & $\begin{array}{l}\text { Caractère } \\
\text { sérieux et } \\
\text { question } \\
\text { nouvelle }\end{array}$ & $\begin{array}{l}\text { Article L. } 243-5 \text { du Code } \\
\text { de la sécurité sociale } \\
\text { (sûretés) }\end{array}$ & $\begin{array}{l}\text { Principe d'égalité : ar- } \\
\text { ticle } 1^{\text {er }} \text { du Préambule } \\
\text { de la Constitution de } \\
\text { 1958, article } 6 \text { de la } \\
\text { DDHC et article } 7 \text { de } \\
\text { la DUDH }\end{array}$ \\
\hline
\end{tabular}


Tableau 4 - Analyse des décisions refusant la transmission de la question prioritaire de constitutionnalité

\begin{tabular}{|c|c|c|c|}
\hline Arrêts & $\begin{array}{l}\text { Motif de non } \\
\text { transmission }\end{array}$ & $\begin{array}{l}\text { Dispositions législatives en } \\
\text { cause }\end{array}$ & Observations \\
\hline $\begin{array}{l}\text { Paris } \\
10 \text { novembre } 2011 \\
n^{\circ} 09 / 03795\end{array}$ & $\begin{array}{l}\text { Absence de } \\
\text { caractère } \\
\text { sérieux }\end{array}$ & $\begin{array}{l}\text { Affiliation au régime } \\
\text { agricole / Article L.311-1 } \\
\text { du Code de la sécurité } \\
\text { sociale }\end{array}$ & \\
\hline $\begin{array}{l}\text { Montpellier } \\
9 \text { octobre } 2019 \\
n^{\circ} 19 / 06518\end{array}$ & $\begin{array}{l}\text { Absence de } \\
\text { caractère } \\
\text { sérieux }\end{array}$ & $\begin{array}{l}\text { Article L.133-4 du Code } \\
\text { de la sécurité sociale }\end{array}$ & \\
\hline $\begin{array}{l}\text { Paris } \\
24 \text { novembre } 2011 \\
n^{\circ} 11 / 07328\end{array}$ & $\begin{array}{l}\text { Absence de } \\
\text { caractère } \\
\text { sérieux }\end{array}$ & $\begin{array}{l}\text { Affiliation régime obliga- } \\
\text { toire / Articles L.611-1 à } \\
\text { L.652-7 et L.722-1 à 723-2 } \\
\text { du Code de la sécurité } \\
\text { sociale }\end{array}$ & \\
\hline $\begin{array}{l}\text { Paris } \\
11 \text { décembre } 2015 \\
n^{\circ} 15 / 18910\end{array}$ & $\begin{array}{l}\text { Disposi- } \\
\text { tions non } \\
\text { applicables } \\
\text { au litige }\end{array}$ & $\begin{array}{l}\text { Articles L.921-4, L.911-1, } \\
\text { L.911-3 et L.911-4 du Code } \\
\text { de la sécurité sociale }\end{array}$ & \\
\hline $\begin{array}{l}\text { Paris } \\
1^{\text {er }} \text { mars } 2018 \\
n^{\circ} 17 / 04051\end{array}$ & & & $\begin{array}{l}\text { Exclusion du corpus } \\
\text { (appel d'une or- } \\
\text { donnance refusant } \\
\text { la QPC relative à } \\
\text { l'article L. 142-2 du } \\
\text { Code de la sécurité } \\
\text { sociale) }\end{array}$ \\
\hline $\begin{array}{l}\text { Douai } \\
21 \text { novembre } 2018 \\
n^{\circ} 18 / 02921\end{array}$ & $\begin{array}{l}\text { Disposi- } \\
\text { tions non } \\
\text { applicables } \\
\text { au litige / } \\
\text { irrecevabi- } \\
\text { lité }\end{array}$ & $\begin{array}{l}\text { Pension de réversion / } \\
\text { Articles L.353-3 et R.354-1 } \\
\text { du Code de la sécurité } \\
\text { sociale }\end{array}$ & \\
\hline $\begin{array}{l}\text { Versailles } \\
28 \text { novembre } 2019 \\
n^{\circ} 17 / 03643\end{array}$ & & & $\begin{array}{l}\text { Exclusion du corpus } \\
\text { (QPC mentionnée } \\
\text { dans la procédure } \\
\text { - QPC ayant fait } \\
\text { l'objet d'un refus de } \\
\text { transmission) }\end{array}$ \\
\hline $\begin{array}{l}\text { Rouen } \\
27 \text { novembre } 2012 \\
n^{\circ} 12 / 04324\end{array}$ & $\begin{array}{l}\text { Absence de } \\
\text { caractère } \\
\text { sérieux }\end{array}$ & $\begin{array}{l}\text { Articles L.611-1 à L.652-7 et } \\
\text { L.722-1 à L.723-24 du Code } \\
\text { de la sécurité sociale }\end{array}$ & \\
\hline
\end{tabular}




\begin{tabular}{|c|c|c|c|}
\hline Arrêts & $\begin{array}{l}\text { Motif de non } \\
\text { transmission }\end{array}$ & $\begin{array}{l}\text { Dispositions législatives en } \\
\text { cause }\end{array}$ & Observations \\
\hline $\begin{array}{l}\text { Versailles } \\
18 \text { octobre } 2018 \\
n^{\circ} 18 / 00068\end{array}$ & $\begin{array}{l}\text { Absence de } \\
\text { caractère } \\
\text { sérieux }\end{array}$ & $\begin{array}{l}\text { Article L.111-1 du Code } \\
\text { de la sécurité sociale }\end{array}$ & \\
\hline $\begin{array}{l}\text { Douai } \\
31 \text { mars } 2016 \\
n^{\circ} 15 / 04600\end{array}$ & $\begin{array}{l}\text { Absence de } \\
\text { caractère } \\
\text { sérieux }\end{array}$ & $\begin{array}{l}\text { Article L.452-4 du Code } \\
\text { de la sécurité sociale }\end{array}$ & \\
\hline $\begin{array}{l}\text { Douai } \\
27 \text { novembre } 2015 \\
n^{\circ} 15 / 03544\end{array}$ & Irrecevable & $\begin{array}{l}\text { Article L.353-1 du Code } \\
\text { de la sécurité sociale }\end{array}$ & Exclusion du corpus \\
\hline $\begin{array}{l}\text { Paris } \\
1^{\text {er }} \text { octobre } 2015 \\
n^{\circ} 14 / 03224\end{array}$ & $\begin{array}{l}\text { Mal fondé } \\
\text { (caractère } \\
\text { non sérieux) }\end{array}$ & $\begin{array}{l}\text { Articles L.2411-1 et L.411-19 } \\
\text { du Code du travail / Ar- } \\
\text { ticle L.114-24 du Code } \\
\text { de la mutualité }\end{array}$ & \\
\hline $\begin{array}{l}\text { Versailles } \\
26 \text { juin } 2014 \\
n^{\circ} 13 / 00011\end{array}$ & $\begin{array}{l}\text { Dépourvu } \\
\text { de carac- } \\
\text { tère sérieux }\end{array}$ & $\begin{array}{l}\text { Article L.323-6, } 4^{\circ} \text { du } \\
\text { Code de la sécurité } \\
\text { sociale }\end{array}$ & \\
\hline $\begin{array}{l}\text { Versailles } \\
16 \text { janvier } 2014 \\
n^{\circ} 13 / 00002\end{array}$ & $\begin{array}{l}\text { Dépourvu } \\
\text { de carac- } \\
\text { tère sérieux }\end{array}$ & $\begin{array}{l}\text { Articles L.161-14 et L.133- } \\
\text { 4-1 du Code de la sécurité } \\
\text { sociale }\end{array}$ & \\
\hline $\begin{array}{l}\text { Versailles } \\
7 \text { février } 2016 \\
n^{\circ} 12 / 00032\end{array}$ & $\begin{array}{l}\text { Dépourvu } \\
\text { de carac- } \\
\text { tère sérieux }\end{array}$ & $\begin{array}{l}\text { Article L.471-1 du Code de } \\
\text { la sécurité sociale dans la } \\
\text { rédaction antérieure à la } \\
\text { loi du } 24 \text { décembre } 2009\end{array}$ & \\
\hline $\begin{array}{l}\text { Versailles } \\
7 \text { février } 2013 \\
n^{\circ} 12 / 00031\end{array}$ & $\begin{array}{l}\text { Dépourvu } \\
\text { de carac- } \\
\text { tère sérieux }\end{array}$ & $\begin{array}{l}\text { Article L.471-1 du Code de } \\
\text { la sécurité sociale dans la } \\
\text { rédaction antérieure à la } \\
\text { loi du } 24 \text { décembre } 2009\end{array}$ & \\
\hline $\begin{array}{l}\text { Versailles } \\
27 \text { janvier } 2011 \\
n^{\circ} 10 / 00019\end{array}$ & $\begin{array}{l}\text { Irrecevabi- } \\
\text { lité car les } \\
\text { dispositions } \\
\text { sont d'ordre } \\
\text { réglemen- } \\
\text { taire }\end{array}$ & $\begin{array}{l}\text { Article L.461-1 du Code } \\
\text { de la sécurité sociale }\end{array}$ & \\
\hline $\begin{array}{l}\text { Versailles } \\
19 \text { mai } 2010 \\
n^{\circ} 19 / 00001\end{array}$ & $\begin{array}{l}\text { Irrecevabi- } \\
\text { lité car les } \\
\text { dispositions } \\
\text { sont d'ordre } \\
\text { réglemen- } \\
\text { taire }\end{array}$ & $\begin{array}{l}\text { Article R.123-3 du Code } \\
\text { de la sécurité sociale } \\
\text { et R.1452-1 du Code du } \\
\text { travail }\end{array}$ & \\
\hline
\end{tabular}




\subsection{Conclusion}

Les explorations dans la base de données d'arrêts civils rendus par les cours d'appel confirment la place importante occupée par les contentieux relatifs à la contestation de l'affiliation aux régimes obligatoires, avec un grand nombre de décisions qui donnent lieu à des amendes civiles et/ou mentionnent un procédé dilatoire. On retrouve en appel le contentieux des "Libérés de la Sécurité sociale » qui a été mentionné par les magistrats des pôles sociaux lors des entretiens et du questionnaire.

Par ailleurs, les décisions de refus de transmission des QPC posées en dehors du contentieux relatif aux cotisations sociales corroborent des observations faites lors des entretiens avec les magistrat'e-s. Certaines questions sont irrecevables du fait du caractère réglementaire des dispositions dont la constitutionnalité est contestée. Par ailleurs, le critère du caractère sérieux sert de filtre laissant une marge d'appréciation relativement importante aux juridictions du fond pour apprécier l'opportunité de transmettre ou non la QPC.

Cette consultation de JuriCA a permis de prendre la mesure de la pratique de la QPC par les justiciables en matière de protection sociale mais aussi de se rendre compte que les décisions QPC rendues constituaient, pour certaines, une véritable source du droit.

\section{B. Les apports de la QPC en droit de la santé lato sensu}

\section{Méthodologie de la recherche}

Mesurer l'apport de la QPC en droit de la santé impose d'abord de dresser un bilan. Il a donc été choisi de faire un bilan thématique des décisions
QPC du Conseil constitutionnel en droit de la santé afin de mesurer non seulement l'apport qualitatif pour la matière mais également l'apport de la QPC pour les justiciables.

Dans le cadre de l'analyse des apports de la QPC en droit de la santé, notre premier et principal objet d'étude a été les décisions QPC du Conseil constitutionnel rendues en la matière depuis la mise en place du dispositif. Toutefois, dès le début de l'élaboration du projet de recherche, nous avons décidé d'élargir le corpus de décisions en y incluant les décisions de non-transmission de la Cour de cassation et du Conseil d'État, qui, lors de l'examen des critères de recevabilité de la question, se font quasi-juges constitutionnels. Enfin, à la suite de notre exploration de la base des décisions des cours d'appel JuriCA, nous avons décidé d'inclure dans le corpus d'analyse, pour chaque décision QPC du Conseil constitutionnel recensée, le nombre de fois où elle avait été citée comme élément du droit positif par une juge devant les juridictions d'appel. En effet, la mise en place du dispositif a suscité un certain nombre d'attentes et a pu être qualifiée de «big-bang juridictionnel $»^{9}$. Il est donc important de déterminer l'apport exact de la QPC au droit matériel. La QPC a-t-elle validé ou au contraire modifié le droit de la santé de manière substantielle ? Quels droits et libertés ont été invoqués et par quel type de justiciable?

Le corpus de décisions analysées a été délimité en adoptant une définition large du droit de la santé, incluant également le droit de la protection sociale et de la santé-travail. En réalité, ce sont trois souscorpus qui ont été constitués.

Dans un premier temps, un recensement exhaustif des décisions QPC rendues a été réalisé à partir du site Internet du Conseil constitutionnel. Il a alors été procédé à une recherche manuelle par mot-clé et à une lecture rapide de l'ensemble des décisions publiées sur le site jusqu'au 31 novembre 2019. Une base de 55 décisions a ainsi été constituée. Les décisions ont fait l'objet d'une analyse

9. Dominique Rousseau, «La question prioritaire de constitutionnalité : un big-bang juridictionnel ? », RDP, $\mathrm{n}^{\circ} 3,2009$, pp. 631 et s. 
sur plusieurs critères : thématique, attribution de mots-clés, mention de la juridiction de renvoi, dispositions législatives en cause, droits et libertés fondamentaux mobilisés, décision du Conseil constitutionnel ainsi que profil du justiciable.

Dans un deuxième temps, les décisions de nontransmission de la Cour de cassation et du Conseil d'État ont été recensées de façon exhaustive et ce jusqu'à la date du 31 novembre 2019. Deux bases ont été constituées selon le même classement que pour celles du Conseil constitutionnel, l'une comportant 107 décisions de non-transmission par la Cour de cassation et l'autre 137 décisions de non transmission par le Conseil d'État.

Dans un troisième temps, au sein de la base $J u r i C A$, il a été procédé au recensement systématique des arrêts qui mentionnent les décisions QPC déjà rendues. Le corpus a été constitué par une requête [ " QPC » sauf « question prioritaire de constitutionnalité » et " code de la sécurité sociale »] afin de conserver le champ matériel du droit de la sécurité sociale et de viser les QPC déjà rendues. Il comprend 1609 arrêts de cours d'appel. Parmi ces 1609 décisions, une recherche systématique des QPC mobilisées a été réalisée, d'abord en utilisant le numéro de décision, puis après lecture des arrêts. Cette analyse a permis de dessiner un tableau des QPC qui ont exercé une influence importante sur le droit de la sécurité sociale, soit en le confortant par la validation des dispositions existantes, soit en y apportant des précisions déterminantes notamment par le biais de réserves d'interprétation. Ces décisions sont mobilisées au même titre que les textes du Code de la sécurité sociale et au même titre que les dispositions législatives auxquelles elles se rapportent.

Pour chaque décision QPC du Conseil constitutionnel, une étude systématique et approfondie a été menée à partir d'une grille d'analyse commune. À l'issue de ces analyses jurisprudentielles, nous disposons d'une vision d'ensemble des apports de la QPC au droit positif en fonction de la thématique et des sujets traités.

\section{Quelques points significatifs ressortant de l'analyse}

L'analyse thématique des décisions QPC du Conseil constitutionnel montre que la fondamentalisation du droit de la santé a touché tous les domaines du droit de la santé et conduit les juges ordinaires à s'intéresser au contentieux constitutionnel.

Le Conseil constitutionnel a ainsi rendu cinq décisions en matière d'organisation, santé publique et sécurité sanitaire, huit en matière d'établissement de santé et de professionnels de santé, treize en matière de bioéthique et de droit des usagers, deux en matière de produits issus du corps humain, de santé et alimentaires, huit en matière de santé-travail, seize en matière de protection sociale et une en matière de santé animale.

Lorsque la QPC est soulevée par un particulier, notamment un salarié, l'analyse des bilans thématiques révèle d'abord une absence : le droit à la santé, garanti par le onzième alinéa du Préambule de la Constitution de 1946, n'est pas le principe fondamental le plus souvent mobilisé par les justiciables. Lorsqu'il l'est, c'est généralement en association avec d'autres principes, notamment le principe d'égalité visé à l'article 6 de la Déclaration des droits de l'homme et du citoyen de 1789. En outre, lorsque la QPC est soulevée par une entreprise, notamment un employeur ou un professionnel de santé, les principes les plus souvent invoqués sont la liberté d'entreprendre, la liberté de commerce, le principe d'égalité devant les charges publiques ou encore le droit de propriété.

De manière générale, le Conseil constitutionnel donne une place importante à la conciliation des droits et libertés fondamentaux avec les raisons d'intérêt général avancées par le législateur au stade de l'adoption des textes. Dans cette conciliation, il fait preuve, notamment en matière de bioéthique, d'un self-restraint certain. En matière de droit de la protection sociale, cela ressort de l'analyse thématique menée et fait également écho aux observations exprimées par les confédérations 
syndicales. Ainsi, dans ce champ, le Conseil constitutionnel décide de façon classique que les différences de traitement sont admises pour autant qu'elles sont justifiées par des raisons d'intérêt général et en rapport direct avec l'objet de la loi. Mais il ne questionne pas pour autant la pertinence actuelle des catégories légales construites par le législateur dans un contexte socio-économique qui a depuis lors changé ; les raisons d'intérêt général sont rarement explicitées dans les décisions (voir p. 104 du rapport).

Les décisions de conformité restent majoritaires tandis que les décisions de non-conformité conduisent le législateur à réviser l'état du droit, parfois de manière très importante comme en matière de soins sans consentement. Quant aux décisions comportant une réserve d'interprétation - ou un effet différé dans le temps --, elles viennent expliciter les règles existantes et constituent ainsi une véritable source du droit.

Les décisions QPC du Conseil constitutionnel qui ont été mobilisées devant les juridictions d'appel comme un moyen de droit s'inscrivent dans quatre thématiques : organisation, santé publique et sécurité sanitaire, santé-travail, bioéthique et droit des usagers, protection sociale. La décision de loin la plus citée est la décision n ${ }^{\circ} 2010-8$ QPC du 18 juin 2010, Époux L., confirmant, avec une réserve d'interprétation importante, la constitutionnalité du régime d'indemnisation des Accidents du Travail et Maladies Professionnelles (AT-MP) en cas de faute inexcusable de l'employeur. Sur un corpus constitué de 1609 arrêts, pas moins de 1208 décisions y font référence. Viennent ensuite la décision $\mathrm{n}^{\circ}$ 2010-78 du 10 décembre 2010, Société IMNOMA, déclarant conforme à la Constitution la composition du Tribunal des affaires de sécurité sociale (TASS) (145 occurrences) et la décision $n^{\circ}$ 2011-127 QPC du 6 mai 2011, Consorts C., relative au régime d'indemnisation des AT-MP des marins (14 occurrences). Au total, sur les 55 décisions QPC du Conseil constitutionnel faisant partie de notre corpus d'analyse en droit de la santé, 10 décisions QPC ont été citées devant les juridictions d'appel avec des fréquences très variables, entre 1 et 1208 fois (tableau 5 ci-dessous).

Tableau 5 - Décisions QPC citées par les arrêts civils de cours d'appel

\begin{tabular}{|c|c|c|c|}
\hline $\begin{array}{l}\text { Décision QPC } \\
\text { citée }\end{array}$ & $\begin{array}{l}\text { Nombre d'occur- } \\
\text { rences parmi les } \\
1609 \text { arrêts du } \\
\text { corpus [QPC] } \\
\text { et [Code de la } \\
\text { sécurité sociale] }\end{array}$ & $\begin{array}{l}\text { Nombre } \\
\text { d'occurrences } \\
\text { parmi un autre } \\
\text { corpus }\end{array}$ & $\begin{array}{l}\text { Décision } \\
\text { du Conseil } \\
\text { constitutionnel }\end{array}$ \\
\hline $\begin{array}{l}\text { Décision n²018- } \\
768 \text { QPC du } 21 \text { mars 2019, } \\
\text { M. Adama S. }\end{array}$ & o & $\begin{array}{l}1 \text { occurrence } \\
\text { parmi les décisions } \\
\text { [QPC] et [Code } \\
\text { civil] }\end{array}$ & $\begin{array}{l}\text { Non-conformi- } \\
\text { té partielle }\end{array}$ \\
\hline $\begin{array}{l}\text { Décision n²016-533 QPC } \\
\text { du } 14 \text { avril 2016, } \\
\text { M. Jean Marc P. }\end{array}$ & o & $\begin{array}{l}3 \text { occurrences avec } \\
\text { la requête [QPC] } \\
\text { et [2016-533] }\end{array}$ & $\begin{array}{l}\text { Conformité } \\
\text { avec réserve } \\
\text { d'interpréta- } \\
\text { tion }\end{array}$ \\
\hline $\begin{array}{l}\text { Décision n²015-500 QPC } \\
\text { du } 27 \text { novembre 2015, So- } \\
\text { ciété Foot Locker France } \\
\text { SAS }\end{array}$ & o & $\begin{array}{l}6 \text { occurrences } \\
\text { parmi les décisions } \\
\text { [QPC] et [Code du } \\
\text { travail] }\end{array}$ & $\begin{array}{l}\text { Non-conformi- } \\
\text { té totale avec } \\
\text { effet différé }\end{array}$ \\
\hline
\end{tabular}




\begin{tabular}{|c|c|c|c|}
\hline $\begin{array}{l}\text { Décision QPC } \\
\text { citée }\end{array}$ & $\begin{array}{l}\text { Nombre d'occur- } \\
\text { rences parmi les } \\
1609 \text { arrêts du } \\
\text { corpus [QPC] } \\
\text { et [Code de la } \\
\text { sécurité sociale] }\end{array}$ & $\begin{array}{l}\text { Nombre } \\
\text { d'occurrences } \\
\text { parmi un autre } \\
\text { corpus }\end{array}$ & $\begin{array}{l}\text { Décision } \\
\text { du Conseil } \\
\text { constitutionnel }\end{array}$ \\
\hline $\begin{array}{l}\text { Décision n²012-235 QPC } \\
\text { du } 20 \text { avril 2012, Associa- } \\
\text { tion Cercle de réflexion et } \\
\text { de proposition d'actions } \\
\text { sur la psychiatrie }\end{array}$ & o & $\begin{array}{l}4 \text { décisions parmi } \\
\text { les décisions } \\
\text { [QPC] et [Code de } \\
\text { la santé publique] }\end{array}$ & $\begin{array}{l}\text { Non-confor- } \\
\text { mité partielle } \\
\text { avec effet } \\
\text { différé }\end{array}$ \\
\hline $\begin{array}{l}\text { Décision n²011-155 QPC } \\
\text { du } 29 \text { juillet 2011, } \\
\text { Mme Laurence } L \text {. }\end{array}$ & o & $\begin{array}{l}1 \text { décision parmi les } \\
\text { décisions [QPC] } \\
\text { et [Code des } \\
\text { pensions civiles et } \\
\text { militaires] }\end{array}$ & Conformité \\
\hline $\begin{array}{l}\text { Décision n²011-127 QPC } \\
\text { du } 6 \text { mai 2011, Consorts C. }\end{array}$ & 14 & & $\begin{array}{l}\text { Conformité } \\
\text { avec réserve }\end{array}$ \\
\hline $\begin{array}{l}\text { Décision n²010-76 QPC } \\
\text { du } 3 \text { décembre 2010, M. } \\
\text { Roger } L .\end{array}$ & 145 & & Conformité \\
\hline $\begin{array}{l}\text { Décision n²010-71 QPC } \\
\text { du } 26 \text { novembre 2010, } \\
\text { Mlle Danielle S. }\end{array}$ & 1 & & $\begin{array}{l}\text { Non-confor- } \\
\text { mité partielle } \\
\text { - effet différé } \\
\text { - réserve }\end{array}$ \\
\hline $\begin{array}{l}\text { Décision n²010-8 QPC } \\
\text { du } 18 \text { juin 2010, Époux L. }\end{array}$ & $\begin{array}{l}1208 \\
\text { avec les erreurs } \\
\text { de plume } \\
1362 \text { occur- } \\
\text { rences }\end{array}$ & & $\begin{array}{l}\text { Conformité } \\
\text { avec réserve }\end{array}$ \\
\hline $\begin{array}{l}\text { Décision n²010- } \\
2 \text { QPC du } 11 \text { juin } 2010 \text {, } \\
\text { Mme Vivianne } L \text {. }\end{array}$ & 1 & & $\begin{array}{l}\text { Non-conformi- } \\
\text { té partielle }\end{array}$ \\
\hline
\end{tabular}

\section{Conclusion}

La décision dont la portée est la plus grande est la décision n ${ }^{\circ}$ 2010-8 QPC du 18 juin 2010, Époux $L$., et ce, à plusieurs égards. D'abord, la réserve d'interprétation figurant dans cette décision sera reprise dans les deux autres décisions rendues à propos de régimes spéciaux, l'un relatif aux salariés travailleurs dans les territoires d'outre-mer ${ }^{10}$, le second relatif aux travailleurs marins ${ }^{11}$. Cette décision pose donc le principe à partir duquel le Conseil constitutionnel a forgé sa jurisprudence à propos du régime d'indemnisation des AT-MP en cas de faute inexcusable de l'employeur. Ensuite,

10. Conseil constitutionnel, décision n $n^{\circ}$ 2016-533 QPC du 14 avril 2016, M. Jean-Marc P. (Accidents du travail - Faute inexcusable de l'employeur : régime applicable dans certaines collectivités d'outre-mer et en Nouvelle-Calédonie).

11. Conseil constitutionnel, décision n ${ }^{\circ}$ 2011-127 QPC du 6 mai 2011, Consorts C. (Faute inexcusable de l'employeur : régime spécial des accidents du travail des marins). 
l'importance de cette décision tient à la place qu'elle occupe désormais dans le contentieux toujours nourri sur cette question. Comme l'a révélé l'examen de la base JuriCA, la décision n ${ }^{\circ} 2010-8$ QPC est devenue une véritable source du droit au même titre que la disposition légale à laquelle elle se rapporte. Ainsi les avocats mais également les juges renvoient de manière explicite et presque systématique à la décision $\mathrm{n}^{\circ}$ 2010-8 QPC sans compter que la réserve d'interprétation du Conseil constitutionnel fait l'objet d'une incorporation dans le Code de la sécurité sociale ${ }^{12}$. C'est sur cette question en particulier que s'est affirmée la capacité de la QPC à devenir une véritable source du droit. D'un point de vue plus substantiel que procédural, la portée de la décision n ${ }^{\circ}$ 2010-8 QPC mérite également d'être soulignée, que ce soit dans le champ du droit de la protection sociale ou dans celui du droit de la responsabilité civile. Dans le champ du droit de la protection sociale, la réserve d'interprétation a en effet conduit les juges du fond à davantage s'intéresser à la nature des préjudices couverts par la réparation forfaitaire prévue par le régime spécial en matière d'AT-MP et à accueillir les demandes en réparation de tous les autres préjudices dont seraient victimes les accidentés du travail et les malades d'origine professionnelle. Cependant, sur ce point, la portée de la décision a été réduite par la Cour de cassation. Par son interprétation de la réserve d'interprétation, la Cour de cassation a limité grandement les effets que pouvait avoir la décision du Conseil constitutionnel du point de vue de la réparation des préjudices subis par les salariés victimes d'AT-MP. Elle retient en effet une interprétation restrictive qui exclut de « l'extension QPC» les préjudices qui sont pris en charge par le livre IV indépendamment du point de savoir si, pour la victime, ces préjudices ont effectivement été pris en charge ${ }^{13}$. Mais c'est plus largement dans le domaine de la responsabilité civile que la décision a eu un retentissement.
Fondée sur l'article 4 de la Déclaration des Droits de l'Homme et du Citoyen, la décision n ${ }^{\circ}$ 2010-8 QPC permet d'affiner les contours du principe de la responsabilité civile, corollaire nécessaire du principe de liberté.

Bien qu'il ne s'agisse pas d'une décision d'inconstitutionnalité ni d'une décision de conformité mentionnant une réserve d'interprétation, la décision $\mathrm{n}^{\circ}$ 2010-76 QPC est également intéressante à mettre en perspective avec les évolutions législatives ultérieures et avec le contentieux dit des «Libérés de la sécurité sociale ». La loi de modernisation de la justice du XXI ${ }^{\mathrm{e}}$ siècle du 18 novembre 2016 a, notamment, réformé l'organisation du contentieux de la sécurité sociale, cette nouvelle organisation ayant subi des "ajustements » ultérieurs via la loi du 23 mars 2019 de programmation 2018-2022 et de réforme pour la justice. Or, tout en renforçant la professionnalisation des assesseurs qui sont désormais soumis à une obligation de formation initiale, le texte a maintenu le principe de l'échevinage. Lors du contrôle de constitutionnalité a priori de ces textes législatifs ${ }^{14}$, l'inconstitutionnalité des dispositions des articles 12 et 95 de la loi du 23 mars 2019 n'a pas été soulevée par les requérants et n'a donc pas été contrôlée. Elle pourrait donc l'être $a$ posteriori par voie de QPC. Cependant, une telle QPC aurait peu de chances de prospérer au regard de la décision $\mathrm{n}^{\circ}$ 2010-76 QPC du 3 décembre 2010, M. Roger L. On peut en effet considérer que la légitimité de la présence d'assesseurs représentant les salariés et les employeurs a été préalablement renforcée par la décision $n^{\circ}$ 2010-76 QPC. En quelque sorte, celle-ci laisse « présumer » la conformité à la Constitution des articles L. 218-1 à 12 du Code de l'organisation judiciaire. En outre, au regard du contentieux dit des «Libérés de la sécurité sociale ", les entretiens avec les magistrats des pôles sociaux et le questionnaire ont

12. On remarquera que le site Légifrance procède de même en visant la décision n ${ }^{\circ}$ 2010-8 QPC sous les articles L. 451-1, L. 452-3 et L. 452-5 du Code de la sécurité sociale.

13. Cass. civ. 2, 30 juin 2011, $n^{\circ} 10-19475$.

14. Conseil constitutionnel, décision $n^{\circ} 2016-739$ DC du 17 novembre 2016, Loi de modernisation de la justice du XXI siècle, et décision n² 2019-778 DC du 21 mars 2019, Loi de programmation 2018-2022 et de réforme pour la justice. 
révélé que la composition du TASS a été spécifiquement contestée par les justiciables dans le cadre de ce mouvement. De fait, cet aspect pourrait donc expliquer les nombreuses occurrences de cette décision dans les arrêts des cours d'appel.

\section{Pistes de réflexion ouvertes et applications envisageables}

En définitive, cette recherche donne un premier aperçu des pratiques et des apports de la question prioritaire de constitutionnalité en droit de la santé, droit de la protection sociale et santé-travail, notamment grâce à l'utilisation de la base de décisions d'appel JuriCA. Elle permet également de dégager plusieurs pistes de réflexion. La création des pôles sociaux au sein des TGI, à la suite de la loi du 18 novembre 2016, emporte intégration des affaires traitées par ces juridictions du premier degré au répertoire général civil (art. 726 du Code de procédure civile). Dès lors, les questions prioritaires de constitutionnalité faisant l'objet d'une codification spécifique, il serait possible dans l'avenir de disposer d'une connaissance précise des caractéristiques de ces affaires et de mieux mesurer les apports de la QPC dans le champ du droit de la protection sociale en première instance.

La QPC a permis de modifier différents aspects du droit de la santé, de la protection sociale et de la santé-travail. En particulier, la réserve d'interprétation de la décision $n^{\circ} 2010-8$ QPC a redessiné les contours du régime d'indemnisation des accidents du travail et maladies professionnelles en cas de faute inexcusable de l'employeur et montre que la QPC peut constituer une véritable source du droit. L'examen a posteriori de la constitutionnalité d'une disposition législative pose toutefois question, notamment au regard du principe de sécurité juridique. Il apparaît par ailleurs que les acteurs pourtant bien institués dans ces domaines du droit ne se saisissent pas toujours de la QPC ou qu'ils le font à l'occasion de la publication des textes d'applications. Par exemple, à propos de la fin de vie, la loi du 2 février 2016 n'a pas été déférée au Conseil constitutionnel. En revanche, l'Union nationale des associations de familles de traumatisés crâniens et de cérébro-lésés a posé une QPC à l'occasion d'un recours en annulation pour excès de pouvoir du décret n²016-1066 du 3 août 2016 précisant les modalités d'organisation des procédures collégiales de décision.

Ainsi, le rôle des organisations syndicales représentatives et des associations d'usagers du système de santé pourrait être renforcé en leur permettant de saisir a priori le Conseil constitutionnel (sur les projets de loi uniquement et dans un délai relativement court). Le Conseil économique, social et environnemental pourrait également se voir confier ce rôle. Au nom des principes de participation des travailleurs et de participation du public, il paraît pertinent d'engager une réflexion sur l'extension de la saisine a priori du Conseil constitutionnel : cela renforcerait le rôle des acteurs défendant des intérêts collectifs, structurés et institutionnalisé et l'effectivité du contrôle de constitutionnalité des lois, sans pour autant porter atteinte au principe de sécurité juridique. 